\title{
Construction of a Plane From an Angular Vector
}

\author{
Yevhen Kharchenko \\ ${ }^{1}$ Zaporizhzhia, Ukraine \\ Correspondence: Yevhen Kharchenko, 14 October 9-174, Zaporizhzhia, 69123, Ukraine. E-mail: Ujin-X@meta.ua
}

Received: February 16, 2020 Accepted: March 13, $2020 \quad$ Online Published: March 18, 2020

doi:10.5539/jmr.v12n2p45

URL: https://doi.org/10.5539/jmr.v12n2p45

\begin{abstract}
The procedure of constructions of a plane from an angular vector in a rectangular coordinate system has been described. The method of creating of an angular vector from two rectilinear vectors has been considered. The property of equality of angular vectors has been described.
\end{abstract}

Keywords: angular vector, cross product of vectors, equality of vectors, plane

\section{Introduction}

With the advent of angular vectors in the vector theory (Kharchenko, 2017), there was a need for their use not only for modeling angular physical quantities, but also for geometric constructions. Angular vectors can be used as oriented planes in the coordinate system. These vectors allow us to perform operations similar to rectilinear vectors, such as: constructing parallel and perpendicular planes; creating a plane perpendicular to the two original planes; construction of a rectilinear vector at the intersection of two planes, etc. One of the first and important steps for these operations is to solve the problem of constructing a plane from an angular vector.

Before deriving a method for constructions of a plane from an angular vector and for a better understanding of the properties of vectors, we will consider:

1. method of creating an angular vector from two rectilinear vectors,

2. property of the equality between angular vectors.

\section{Creating an Angular Vector}

The equation linking rectilinear and angular vectors is the cross product equation $\bar{a} \times \bar{b}=\widehat{c}$. This equation shows not only the relations between vectors, but also the method of creating an angular vector from two rectilinear vectors.

The equation shows that an angular vector is created from a sequence of two rectilinear (perpendicular to each other) vectors. The sequence of rectilinear vectors determines the direction of an angular vector.

In geometric form, when creating an angular vector for the equation $\bar{a} \times \bar{b}=\widehat{c}$, it is convenient to imagine that rectilinear vector begins to be created $\bar{b}$ at the end of the rectilinear vector $\bar{a}$. And the position of these rectilinear (perpendicular) vectors, sets the direction of the angular vector $\widehat{c}$, Fig. 1.

The direction sign of an angular vector, determines the angular direction in the selected coordinate system, just like the directions of the coordinate axes in the coordinate system determine the sign of rectilinear vector.

The value of an angular vector is equal to the area of the rectangle formed from rectilinear vectors.

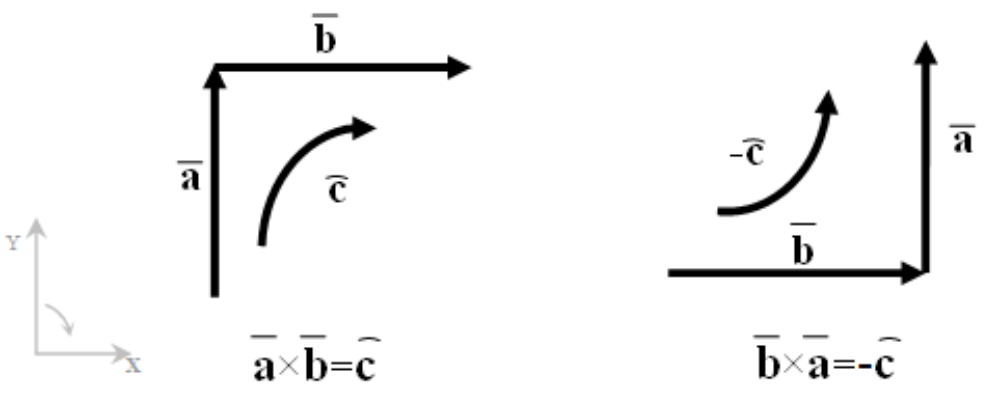

Figure 1. Creating an angular vector of two rectilinear vectors 
The same angular vector can be created from different combinations of rectilinear vectors in the cross product, Fig. 2 .

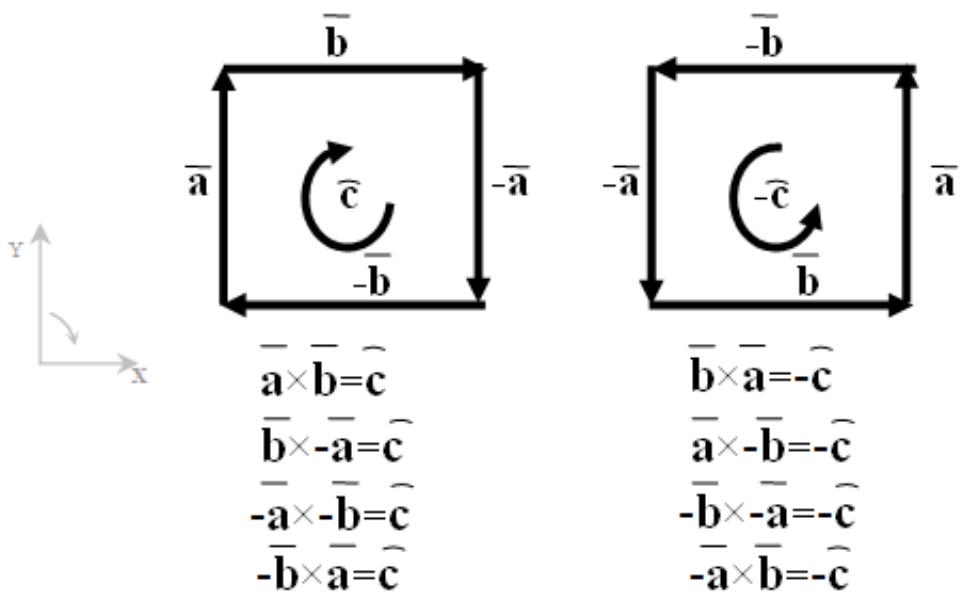

Figure 2. Combinations of cross product of vectors

The choice (or derivation) of the equation for finding the projections of an angular vector in the coordinate-vector form depends on the selected coordinate system. These equations also depend on the sequence of rectilinear vectors in the cross product.

For all variants of rectangular coordinate systems (and there are 96 types $=8$ octants of dividing the space $* 6$ variants of the location of the axis names in each octant * 2 variants of the angular direction for each version of the coordinate system), only two versions of the equations for finding the projections of angular vectors can be derived.

For a positive angular direction between the axes $O y \rightarrow O x \rightarrow O z$ (the direction can be selected both clockwise and counterclockwise, and depends on the selected coordinate system):

$$
\begin{array}{r}
\hat{c}=\bar{a} \times \bar{b}=\left(a_{y} b_{x}-a_{x} b_{y}\right) \hat{l}+\left(a_{z} b_{y}-a_{y} b_{z}\right) \hat{m}+\left(a_{x} b_{z}-a_{z} b_{x}\right) \hat{n}=c_{x y} \hat{l}+c_{y z} \hat{m}+c_{z x} \hat{n} \\
c_{x y}=a_{y} b_{x}-a_{x} b_{y} \quad c_{y z}=a_{z} b_{y}-a_{y} b_{z} \quad c_{z x}=a_{x} b_{z}-a_{z} b_{x}
\end{array}
$$

For a positive angular direction between the axles $O x \rightarrow O y \rightarrow O z$ (the direction can be selected both clockwise and counterclockwise, and depends on the selected coordinate system):

$$
\begin{aligned}
\hat{c} & =\bar{a} \times \bar{b}=\left(a_{x} b_{y}-a_{y} b_{x}\right) \hat{l}+\left(a_{y} b_{z}-a_{z} b_{y}\right) \hat{m}+\left(a_{z} b_{x}-a_{x} b_{z}\right) \hat{n}=c_{x y} \hat{l}+c_{y z} \hat{m}+c_{z x} \hat{n} \\
c_{x y} & =a_{x} b_{y}-a_{y} b_{x} \quad c_{y z}=a_{y} b_{z}-a_{z} b_{y} \quad c_{z x}=a_{z} b_{x}-a_{x} b_{z}
\end{aligned}
$$

This option of coordinating the direction of angular vectors (and their projections) in various coordinate systems is slightly different from that proposed earlier in "Angular Vectors in the Theory of Vectors". This description is the best, since it completely solves the problem of choosing the angular direction in the coordinate system and the equations for finding projections of an angular vector.

For the cross product equation $\bar{a} \times \bar{b}=\widehat{c}$, you do not need to use the right-hand rule (in mathematics) and the right-hand screw rule (in theoretical mechanics), which greatly simplifies the solution of problems and increases the choice of the coordinate system.

\section{Property of the Equality Between Angular Vectors}

Let's remember that two rectilinear vectors $\bar{a}, \bar{b}$ are equal when they:

- have equal modules,

- are equally oriented relative to the coordinate axes (they have the same projection values on the corresponding coordinate axes),

- directions of vectors coincide (signs of all projections of the vectors on coordinate axes coincide).

When writing of rectilinear vectors in algebraic form, vectors are equal $\bar{a}\left(a_{x}, a_{y}, a_{z}\right)=\bar{b}\left(b_{x}, b_{y}, b_{z}\right)$ when their projections $a_{x}=b_{x}, a_{y}=b_{y}, a_{z}=b_{z}$ are equal.

The equality of angular vectors $\hat{c}, \hat{d}, \hat{t}$ is very similar to the properties of rectilinear vectors:

- they have equal modules,

- they are equally oriented relative to the coordinate axes (they have the same projection values on the corresponding 
coordinate planes),

- their directions of vectors coincide.

When angular vectors are written in algebraic form, vectors are equal $\hat{c}\left(c_{x y}, c_{y z}, c_{z x}\right)=\hat{d}\left(d_{x y}, d_{y z}, d_{z x}\right)=\widehat{t}\left(t_{x y}, t_{y z}, t_{z x}\right)$ when their projections are equal:

$$
c_{x y}=d_{x y}=t_{x y}, \quad c_{y z}=d_{y z}=t_{y z}, \quad c_{z x}=d_{z x}=t_{z x} .
$$

But, despite the similar properties of the equality of rectilinear and angular vectors, there are differences between them. They appear during the geometric construction of vectors, Fig. 3.

Equal rectilinear vectors are always parallel and codirectional in space (coordinate system).

For equal angular vectors, only the planes in which they are located are parallel. But the location of an angular vector in the plane (the location of the rectilinear vectors from which an angular vector is built) can be any. And the shape of the resulting the area figure (rectangles or squares) can be any. Moreover, the areas of equal angular vectors are equal $S(\widehat{c})=S(\hat{d})=S(\widehat{t})=|\hat{c}|=|\widehat{d}|=|\hat{t}|$ to the modules of angular vectors.

From the equality of angular vectors it follows that the same angular vector can be represented from various combinations of rectilinear vectors. Therefore, angular vectors are often displayed conditionally (without a geometric figure).

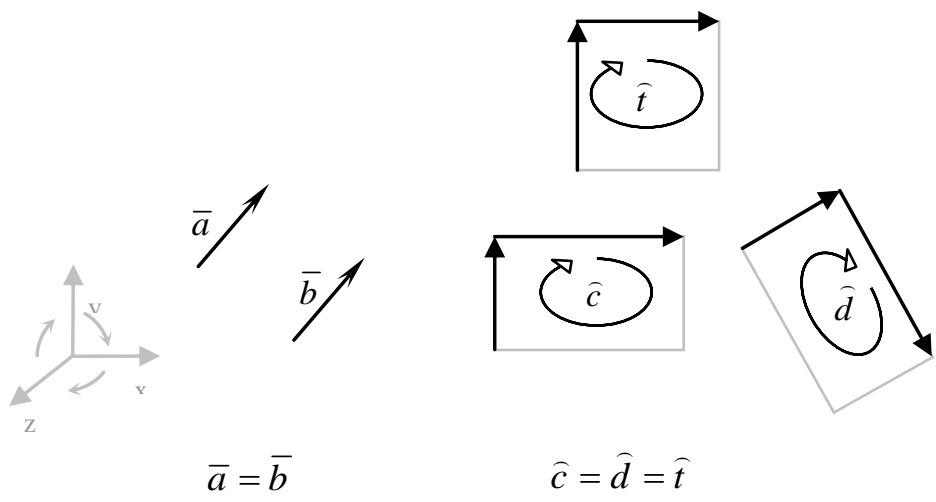

Figure 3. Equality of rectilinear and angular vectors

It also follows from the equality of angular vectors that the projection of an angular vector onto any other plane (including the coordinate one) does not depend on the shape and location of an angular vector on the plane in which it is located.

\section{The Method of Construction of a Plane From an Angular Vector}

It is difficult to construction of an angular vector and its projections onto the coordinate planes in a coordinate system. After all, we only have the area values of the figures on these planes (projections of an angular vector), and we do not know their shapes and locations in the coordinate planes. And to construction of them, we need the original two rectilinear vectors. Their location, size, and direction determine the location, direction, and shape of an angular vector.

Based on the property of the equality of angular vectors, we can represent an angular vector from any sequence of two rectilinear vectors.

Let's imagine that the angular vector $\widehat{c}\left(c_{x y}, c_{y z}, c_{z x}\right)$ is construction of from two rectilinear vectors $\bar{a}\left(a_{x}, a_{y}, 0\right)$ and located in the coordinate planes, Fig. 4 . These vectors begin and end on the coordinate axes and construction of a plane in the form of a triangle in the coordinate system. Let's find the coordinates of the points $A\left(x_{A}, 0,0\right), B\left(0, y_{B}, 0\right)$, $C\left(0,0, z_{C}\right)$, creating this plane.

As you can see, the vectors $\bar{a}, \bar{b}$ are not perpendicular. But this is not important because the cross product $\bar{a} \times \bar{b}=\widehat{c}$ takes into account the location of the original rectilinear vectors. Only part of one of the vectors that can be represented perpendicular to the second vector will participate in the equation.

To find the projections of rectilinear vectors $\bar{a}, \bar{b}$, we need the condition $a_{x}= \pm b_{x}$. This condition determines the dependence of the projection values of two vectors $\bar{a}, \bar{b}$, which allow them to be placed so that they begin and end on the coordinate axes, and the signs allow us to solve the radical expression (3). 


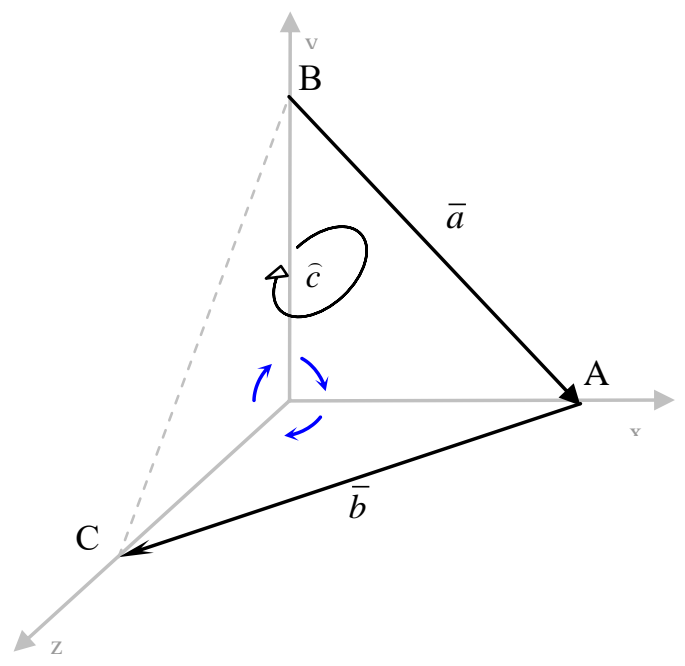

Figure 4. Construction of a plane in the form of a triangle

For the coordinate system with an angular direction $O Y \rightarrow O X \rightarrow O Z$, we find the equation for finding the projections of the angular vector from the cross product equation (1).

$$
\begin{aligned}
& c_{x y}=a_{y} b_{x}-a_{x} b_{y} \\
& c_{y z}=a_{z} b_{y}-a_{y} b_{z} \\
& c_{z x}=a_{x} b_{z}-a_{z} b_{x}
\end{aligned}
$$

We find the projections of rectilinear vectors from these equations.

$$
\begin{array}{lll}
c_{x y}=a_{y} \cdot b_{x}-a_{x} \cdot 0 & c_{y z}=0 \cdot 0-a_{y} \cdot b_{z} & a_{x}=\frac{c_{z x}}{b_{z}}=\mp \frac{c_{z x} \cdot c_{x y}}{c_{y z} \cdot a_{x}} \\
a_{y}=\frac{c_{x y}}{b_{x}}=\frac{c_{x y}}{ \pm a_{x}} \quad, \quad b_{z}=\frac{c_{y z}}{-a_{y}}=-\frac{c_{y z} \cdot b_{x}}{c_{x y}}=\mp \frac{c_{y z} \cdot a_{x}}{c_{x y}} & a_{x}=\sqrt{\mp \frac{c_{z x} \cdot c_{x y}}{c_{y z}}}
\end{array}
$$

For the coordinate system with an angular direction $O X \rightarrow O Y \rightarrow O Z$, the equation for finding the projections of the angular vector is as follows:

$$
\begin{aligned}
& c_{x y}=0-a_{y} \cdot b_{x} \quad c_{y z}=a_{y} \cdot b_{z}-0 \\
& a_{y}=-\frac{c_{x y}}{b_{x}}=\frac{c_{x y}}{\mp a_{x}}, \quad b_{z}=\frac{c_{y z}}{a_{y}}=-\frac{c_{y z} \cdot b_{x}}{c_{x y}}=\mp \frac{c_{y z} \cdot a_{x}}{c_{x y}}, \quad a_{x}=\sqrt{ \pm \frac{c_{z x} \cdot c_{x y}}{c_{y z}}}
\end{aligned}
$$

The choice of the upper or lower sign in the three equations for (3), (4) depends on whether the radical $\boldsymbol{a}_{\boldsymbol{x}}$ is a real number (whether the radical expression is positive). If there are signs in the projections of an angular vector $\bar{c}(-,-,-)$, $\hat{c}(-,+,+), \hat{c}(+,-,+), \hat{c}(+,+,-)$, the "minus" sign is selected in the subradical equation. If there are signs in the projections of an angular vector $\hat{c}(+,+,+), \widehat{c}(-,-,+), \widehat{c}(+,-,-), \widehat{c}(-,+,-)$, then the "plus" sign is selected. Accordingly, the upper or lower sign in the equations $(3,4)$ of finding the other two projections of rectilinear vectors is selected.

The radical solution for the projection $a_{x}$ gives two solutions. As a result, we get two pairs of oppositely directed vectors $\bar{a}, \bar{b}$ and $-\bar{a},-\bar{b}$ located on the same plane. 
In order to find a pair of vectors, we used the cross product equation $\bar{a} \times \bar{b}=\widehat{c}$. In fact, we do not know the sequence of rectilinear vectors in the cross product that suits us (for the same angular vector $\bar{c}$ ). If we chose the cross product $\bar{b} \times \bar{a}=\bar{c}$, we would get pairs of vectors: $\bar{b},-\bar{a} ;-\bar{b}, \bar{a}$.

Let's choose from the obtained four vectors a suitable pair of vectors by an additional condition $a_{x}=-b_{x}$.

Next, we find the coordinates of points A, B, C.

$$
\begin{array}{llll}
\multicolumn{1}{c}{\bar{a}=\left(x_{B}-x_{A}, y_{B}-y_{A}, 0\right) ;} & \bar{b}=\left(x_{A}-x_{C}, 0, z_{A}-z_{C}\right) ; \\
x_{B}-x_{A}=a_{x} & y_{B}-y_{A}=a_{y} & x_{A}-x_{C}=b_{x} & z_{A}-z_{C}=b_{z} \\
x_{B}=0 & y_{A}=0 & x_{C}=0 & z_{A}=0 \\
x_{A}=-a_{x} & y_{B}=a_{y} & x_{A}=b_{x} & z_{C}=-b_{z} \\
& A\left(x_{A}, 0,0\right) ; & B\left(0, y_{B}, 0\right) ; & C\left(0,0, z_{C}\right)
\end{array}
$$

Example 1. Construction of a plane from an angular vector.

Let us be given an angular vector $\widehat{c}(10,-5,8)$ and a coordinate system, Fig. 5. It is necessary to find points $A\left(x_{A}, 0,0\right)$, $B\left(0, y_{B}, 0\right), C\left(0,0, z_{C}\right)$ on the coordinate axes to construct a plane in which the angular vector is located.

For our condition: $\widehat{c}(10,-5,8)$ and with a positive angular direction $O Y \rightarrow O X \rightarrow O Z$, we choose the upper sign for equations (3).

$$
a_{x}=\sqrt{-\frac{c_{z x} \cdot c_{x y}}{c_{y z}}}=\sqrt{-\frac{8 \cdot 10}{-5}}= \pm 4, \quad a_{y}=\frac{c_{x y}}{b_{x}}=\frac{c_{x y}}{+a_{x}}=\frac{10}{ \pm 4}= \pm \frac{5}{2}, \quad b_{z}=-\frac{c_{y z} \cdot a_{x}}{c_{x y}}=-\frac{-5 \cdot( \pm 4)}{10}= \pm 2,
$$

And we get two pairs of rectilinear vectors:

$$
\bar{a}_{1}\left(4, \frac{5}{2}, 0\right), \quad \bar{b}_{1}(4,0,2) ; \quad \bar{a}_{2}\left(-4,-\frac{5}{2}, 0\right), \quad \bar{b}_{2}(-4,0,-2)
$$

Each pair of rectilinear vectors obtained can be used to construct an angular vector. But we, by the condition of the problem, need to construction of a plane with points located on the coordinate axes. To do this, we can re-find rectilinear vectors using the cross product $\bar{b} \times \bar{a}=\bar{c}$ and get the pairs of vectors

$$
\bar{b}_{2}(-4,0,-2), \bar{a}_{1}\left(4, \frac{5}{2}, 0\right) ; \quad \bar{b}_{1}(4,0,2), \bar{a}_{2}\left(-4,-\frac{5}{2}, 0\right) .
$$
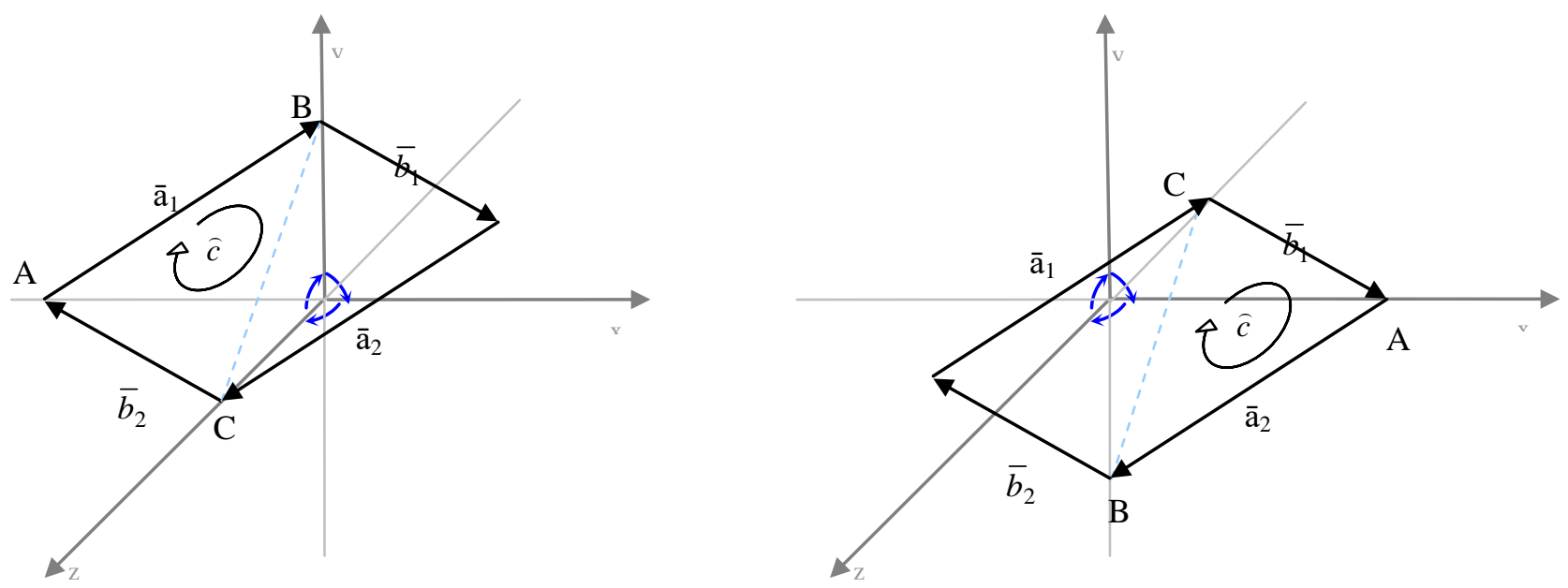

Figure 5. Construction of a plane from angular vector

Or, we choose a pair $\bar{a}, \bar{b}$, satisfying the condition $a_{x}=-b_{x}$ from four vectors 


$$
\bar{a}_{1}\left(4, \frac{5}{2}, 0\right), \bar{b}_{2}(-4,0,-2), \quad \text { then } \quad x_{A}=-a_{x}=b_{x}=-4, \quad y_{B}=a_{y}=\frac{5}{2}, \quad z_{C}=-b_{z}=2
$$

Answer 1: $\quad A(-4,0,0) ; B\left(0, \frac{5}{2}, 0\right) ; C(0,0,2)$

Or $\quad \bar{a}_{2}\left(-4,-\frac{5}{2}, 0\right), \bar{b}_{1}(4,0,2), \quad$ then $\quad x_{A}=-a_{x}=b_{x}=4, \quad y_{B}=a_{y}=-\frac{5}{2}, \quad z_{C}=-b_{z}=-2$

Answer 2: $\quad A(4,0,0) ; B\left(0,-\frac{5}{2}, 0\right) ; C(0,0,-2)$

Conclusion: Both solutions are fair and create two parallel planes.

Example 2. Construction of a plane passing through a point from an angular vector.

If the angular vector $\widehat{c}(10,-5,8)$ from the previous example passes through a point $D(2,5,-3)$, it is quite easy to construction of a plane from any pair of vectors $\pm \bar{a}, \pm \bar{b}$. The point $D$ belongs to two vectors and can be the beginning or end of each vector. Let's find the coordinates of the points $T, P$ located on the desired plane.

Let the point $D$ be at the beginning of the vector $\bar{a}_{1}\left(4, \frac{5}{2}, 0\right)$, then the coordinates of the point $T\left(x_{T}, y_{T}, z_{T}\right)$ at the end of the vector are as follows:

$$
\begin{gathered}
T\left(x_{T}=a_{x}+x_{D}, y_{T}=a_{y}+y_{D}, z_{T}=a_{z}+z_{D}\right) \\
T\left(x_{T}=4+2, y_{T}=\frac{5}{2}+5, z_{T}=0-3\right) \\
T\left(6, \frac{15}{2},-3\right)
\end{gathered}
$$

Let the point $D$ be at the beginning of the vector $\bar{b}_{1}(4,0,2)$, then the coordinates of the point $P\left(x_{P}, y_{P}, z_{P}\right)$ at the end of the vector are as follows:

$$
\begin{gathered}
P\left(x_{P}=b_{x}+x_{D}, y_{P}=b_{y}+y_{D}, z_{P}=b_{z}+z_{D}\right) \\
P\left(x_{P}=4+2, y_{P}=0+5, z_{P}=2-3\right) \\
P(6,5,-1)
\end{gathered}
$$

Answer: It is one of the options for creating a plane through points $D(2,5,-3), T\left(6, \frac{15}{2},-3\right), P(6,5,-1)$

\section{Conclusion}

The described simple procedure shows that construction of a plane from an angular vector allows us to find several solutions at once (to create several parallel planes) that expand the capabilities of engineers in geometric constructions.

The considered method of forming an angular vector from two rectilinear vectors shows a geometric relationship between these vectors. This explanation of the logical dependence of rectilinear and angular vectors is the missing link in proving the existence of the cross product equation for vectors $\bar{a} \times \bar{b}=\bar{c}$.

The considered property of the equality of angular vectors allows us to expand the methods of solving problems and when solving them, visually represent the behavior of the angular vector and its projections.

\section{Refutation}

In the paper "Angular Vectors in the Theory of Vectors", the projections of an angular vector onto the coordinate planes were connected by a sequence of indices

$$
b_{x y}=-b_{y x}, \quad b_{y z}=-b_{z y}, \quad b_{z x}=-b_{x z} .
$$


This rule will be superfluous, because it adds excessive attention to the indexes, and the positive direction of the projections of an angular vector is determined by the selected coordinate system in which there is an angular direction.

The sequence of indices should not affect the direction of the projection of an angular vector.

\section{References}

Amel'kin, N. I. (2000). Kinematics and dynamics of a rigid body. M.: MFTI. (Russian)

Andraphanova, N. V. (2015). Geometrical similarity transformations in dynamic geometry environment GEOGEBRA. European Journal of Contemporary Education, 12(2), 116-128. https://doi.org/10.13187/ejced.2015.12.116

Baumgart, B. G. (1974). Geometric modeling for computer vision (No. STAN-CS-74-463). STANFORD UNIV CA DEPT OF COMPUTER SCIENCE.

Dorst, L., Fontijne, D., \& Mann, S. (2010). Geometric algebra for computer science: an object-oriented approach to geometry. Elsevier.

Faas, F. G., \& van Vliet, L. J. (2003, June). 3D-orientation space; filters and sampling. In Scandinavian conference on image analysis (pp. 36-42). Springer, Berlin, Heidelberg. https://doi.org/10.1007/3-540-45103-X_6

Glynn, D. G. (2010). Theorems of points and planes in three-dimensional projective space. Journal of the Australian Mathematical Society, 88(1), 75-92. https://doi.org/10.1017/S1446788708080981

Kharchenko, Y. M. (2017). Angular Vectors in the Theory of Vectors. Journal of Mathematics Research, 9(5), 71. https://doi.org/10.5539/jmr.v9n5p71

Kharchenko, Y. M. (2019). Unresolved problems in mechanics using vectors. 20th International Scientific and Technical Conference "The Progressive Technics, Technology and Engineering Education". Ukraine. Kherson. NTUU "KPI-Sikorsky", 42-45. (Ukrainian). Retrieved from http://conf.mmi.kpi.ua/proc/article/view/180905/180895

Kortenkamp, U. H., \& Richter-Gebert, J. (1998). Geometry and Education in the Internet Age.

Maresch, G. (2014). Strategies for Assessing Spatial Ability Tasks. Journal for Geometry and Graphics, 18, 125-132.

Marinkovic, V., Janicic, P., \& Schreck, P. (2014). Solving geometric construction problems supported by theorem proving. In Proceedings of the 10th International Workshop on Automated Deduction in Geometry (ADG 2014) (pp. 121-146).

Pottmann, H., Liu, Y., Wallner, J., Bobenko, A., \& Wang, W. (2007). Geometry of multi-layer freeform structures for architecture. ACM Transactions on Graphics (TOG), 26(3), 65. https://doi.org/10.1145/1276377.1276458

Schreck, P. (2001, July). Robustness in cad geometric constructions. In Proceedings Fifth International Conference on Information Visualisation (pp. 111-116). IEEE.

Wallner, J., Krasauskas, R., \& Pottmann, H. (2000). Error propagation in geometric constructions. Computer-Aided Design, 32(11), 631-641. https://doi.org/10.1016/S0010-4485(00)00053-1

Wang, K., Tong, Y., Desbrun, M., \& Schröder, P. (2006). Edge subdivision schemes and the construction of smooth vector fields. ACM Transactions on Graphics (TOG), 25(3), 1041-1048. https://doi.org/10.1145/1141911.1141991

Zadorozhnyj, V. N. (2010). Higher mathematics for technical universities. Part II. Analytic geometry: Tutorial. Tomsk. (Russian).

\section{Copyrights}

Copyright for this article is retained by the author(s), with first publication rights granted to the journal.

This is an open-access article distributed under the terms and conditions of the Creative Commons Attribution license (http://creativecommons.org/licenses/by/4.0/). 